\title{
Making Sense of Communication Associated with Artifacts during Early Design Activity
}

\author{
Moushumi Sharmin and Brian P. Bailey \\ University of Illinois at Urbana-Champaign, 201 N Goodwin Avenue, Urbana, IL 61801, USA \\ \{sharmin2, bpbailey\} @illinois.edu
}

\begin{abstract}
Communication associated with artifacts serves a critical role in the creation, refinement, and selection of conceptual ideas. Despite the close relationship between ideas and surrounding communication, effective integration ofthese two types of design materials are not well-supported by exiting design tools - resulting in ad-hoc and ineffective strategies for managing communication during the design process. In this paper, we report the results of a contextual inquiry $(\mathrm{N}=15)$ aimed at understanding communication practices, its role in the design process, and strategies utilized by designers to manage and utilize communication outcomes in relation to artifacts. Our findings show thatmore than $50 \%$ of early design activity consists of three categories of communication (information seeking, brainstorming, and feedback) and communication practice varies as a function of expertise, organizational and social factors. Additionally, novice and freelance designers exhibit greater reliance on online forums to find suitable communication partners to generate and refine ideas whereas experts communicate with other experts or team members for information collection and sharing.
\end{abstract}

Keywords: Design, Artifacts, Communication, Ideation, User Study.

\section{Introduction}

Communication is a central part of the ideation process, especially for creative, nonroutine design activity [1, 2]. Many types of communication (e.g., client correspondence, brainstorm discussions, and feedback on initial ideas) influence the ideation process. Research showsthat discussion associated with artifacts promotes design thinking [1], enables generation and refinement of alternatives [3], and contextualizes the design process [4]. In this paper, we use the term ideation processto refer to activities associated with the generation and evaluation of design artifacts during the early design phases. In addition, we use the term design communication to refer toanytype of exchange, verbal (face-to-face, over phone) or written (e-mail, IM, etc.), synchronous (phone) or asynchronous (e-mail) related to ideation activities.

Research on design has underscored the importance of communication in the design process [27], examining it in specific situations such as studying communication roles [8] and negotiation strategies $[2,8]$ within co-located teams [9, 27]or specific types of communication such as rationale related to design choices [10, 11$]$. Research on collaborative and participatory design focused on the importance of 
communication among stakeholders [27]. Research aiming to support early design activities has concentrated on how designers collect, construct, and manage artifacts $[6,7]$, but has paid less attention to the inter-connection between artifacts and communication.Little emphasis has been given to the integration of artifacts and communication in design tools, forcing designers to invest significant effort to capture and access the connections between them. Research on design reuse has also identified this lack of connection as one of the main barriers for supporting reuse[19].

In this paper, we report resultsfrom a contextual inquiry investigating the role of communication surrounding artifacts during the ideation process. We examine details of designers'communication practice, focusing on communication partners, when in the design process communication occurs, the communication channels utilized, and how the outcomes of communication are captured and utilized in the design process. We also probe social and organizational factors that influence designers' communication behavior. Based on our lessons, we propose guidelines to better integrate communication within existing and future design tools.

Our study consisted of semi-structured interviews with professional designers in the creative design domains. We selected designers having different design backgrounds (graphic and industrial)and working in different settings (design firm and freelancers) to identify similarities and differences in their communication practices. We also studied designers having varying levels of expertise (between 1 to 33 years).Our findings show that communication accounts for more than $50 \%$ of ideation activity. Designers utilize communication to seek information,generate, refine, and select ideas, and to resolve creative blocks. While work setting and expertise influence designers' communication practice, all designers struggled to capture and connect communication outcomes with relevant artifacts. The main contribution of this work is providing new understanding of communication practices related to the use of artifacts across domains, work settings, and levels of expertise and a set of actionable implications for designing better design support tools.

\section{Related Work}

\subsection{Studies of Design Communication}

The role of communication is well researched in the realm of collaborative and distributed work $[12,13,14]$. Kraut et al. studied informal communication as a means for improving collaboration and coordination in an organizational setting [13], while Chiu examined the effect of team organization on communication [12]. Sonnenwald examined communication as a collaboration tool for multi-disciplinary teams [2]. This thread of research focused on communication as a means for improving collaboration efficiency. During the ideation process, the role of communication transcends collaboration efficiency, as it allows designers to learn about the design space and guides the generation, selection, and refinement of artifacts. Communication during ideation often includes artifacts such as sketches, drawings, prototypes, and other visuals [1]. Studies focusing on communication therefore offer only a partial picture of the ideation process, ignoring the influence of communication outcomes in the generation and refinement of artifacts. One consequence is that existing tools do not provide adequate 
support for integration of communication and related artifacts; making it difficult to utilize them during ideation. We aim to fill this gap.

Design researchers have attended to formal and organized communication (e.g., brainstorming sessions) $[15,16]$. Eckert and Stacey conducted several studies investigating communication in collaborative design tasks and offered six broad dimensions of communication, including form of communication, task, subject and tool expertise, and objectives for communication [15]. Stempfle and Badke-Schaub focused on design thinking as a team process and viewed communication as a representation of individual and team thinking [16]. Our research extends prior work by probing the various underlying reasons that motivate and influence communication associated with artifacts in practice.

\subsection{Studies of Early Stages of Creative Design Practice}

Research on early phases of creative design has focused on different types of artifacts and how these artifacts (e.g., sketches and prototypes) impact ideation process [17, 18]. Bonnardel studied the influence of information availability on the idea generation process[4]. Researchers also studied the impact of capturing, accessing, and reusing artifacts in the ideation process[19]. A separate thread of research centered on the creation andutilization of sketches and early prototypes to externalize ideas[7, 20]. Researchers also focused on facilitating ideation process by capturing the history of the progression of artifacts [21]. Additionally, researchers have focused on the influence of workspace activities within design teams during the artifact generation process [27].

Prior research on early stages of creative design has emphasized the construction of artifacts, especially through sketching, without regard for the rich communication that influenced and was prompted by those artifacts. Researchers consider communication to be an inseparable part of the ideation process [27] as it not only guides the design process, but also allows designers to represent and support their design decisions [1]. Studying artifacts provides only a partial understanding of the early design process, as it fails to portray thought processes, discussions that prompted changes in artifacts, and decisions that led to the evolution of the ideas. We aim to offer better understanding of the relationship between artifacts and related communication by studying designers' communication practices.

\subsection{Systems Supporting Design Communication}

Most communication support systems focus on capturing decisions and deliberations related to design problems and are aimed to support redesign and maintenance issues $[10,22,23]$. Another related class of system supports communication among stakeholders largely by capturing decisions surrounding artifact design [24, 25]. Mood boards and similar systems often capture design scenarios, explored alternatives, and early design activity to convey ideas and design choices to clients [5,26]. These systems try to provide awareness of ongoing design activity by capturing and representing artifacts or specific types of communication (e.g. rationale), but ignore the relationship between artifacts and other types of communication (e.g., discussions, feedback, and client preferences) that shapes the ideation process. The resulting systems thus fail to represent the design process, making it difficult for designers to 
utilize and understand the process in which the artifacts were created[19]. We believe research aimed at understanding designers' communication practice in relation to artifactscan provide valued insight and guide the design of tools that would provide more effective management of the design process.

\section{Methodology}

The goal of our study was to understand communication practices during ideation activities holistically, investigate designers' motivations and strategies for initiating, managing, and utilizing communication outcomes, and examine organizational and socialfactors that influence their communication behavior. Our study consisted of semi-structured interviews with professional designers with varying levels of expertise and coming from different creative design domains. We used self-report data to classify designers as expert and novice.For example, one designer has a total of three years of professional experience and rated his expertise level as "novice."Another designer has more than 10years of professional design experience and rated herself as an "expert." We also considered two types of work setting, designers who work in an organizational setting (design firms, $\mathrm{N}=8$ ) and designers who work independently (freelance, $\mathrm{N}=7$ ). See table 1 for background of the study participants.

The interview had 19 questions guided by our prior research on reuseof existing design knowledge during the early stages of creative design domains[19].Table 2 lists sample questions asked during the interview.We began the interview by asking the designer to briefly describe a recent or ongoingproject and encouraged her to share stories about communication practices during their ideation process to ground the discussion. For example, one designer was working on the design of a social networking site for a corporate setting. During the interview, the designer focused on how communication with clients helped to accumulate various types of informationneeded for designing the site and how discussion with other designers helped to refine his early ideas.

Table 1. Background of study participants

\begin{tabular}{|l|l|l|l|l|l|}
\hline Domain & \multirow{2}{*}{$\begin{array}{l}\text { Number of } \\
\text { Participant }\end{array}$} & \multicolumn{2}{|c|}{ Work Setting } & \multicolumn{2}{|c|}{ Level of Expertise } \\
\cline { 3 - 6 } & & $\begin{array}{l}\text { Design } \\
\text { Organization }\end{array}$ & Freelance & Expert & Novice \\
\hline Graphic & 7 (3 female) & 5 & 2 & 4 & 3 \\
\hline Web & 4 & 1 & 3 & 2 & 2 \\
\hline Industrial & 4 (2 female) & 2 & 2 & 2 & 2 \\
\hline
\end{tabular}

Interviews lasted no more than two hours and interviewees received $\$ 20$ for their participation.Ten of these interviews were conducted in the designers' workspace and the remaining via phone due to distance. While phone interviews limited our ability to directly examine the workspace, we requested the interviewees to share additional materials such as photos of the workspace, screen-captures of the artifact and communication management systems, and records of captured communication with us to gain better understanding of their communication practice. 
Table 2. Sample of the questions asked during an interview

\begin{tabular}{|l|}
\hline Types of Communication \\
\hline What are the different types of communication that occur throughout the design \\
process? \\
\hline Frequency and Motivation \\
\hline $\begin{array}{l}\text { How often do you communicate with other designers during the early design phase? } \\
\text { What motivates you to do so? }\end{array}$ \\
\hline Capture and Access \\
\hline $\begin{array}{l}\text { Why and how do you capture and access communications for an ongoing or a prior } \\
\text { project, if at all? What motivates you to do so? }\end{array}$ \\
\hline Medium of Communication \\
\hline $\begin{array}{l}\text { Which is your preferred method for communication during early design (face-to-face, } \\
\text { e-mail, etc.)?Why do you prefer this method over the other methods? }\end{array}$ \\
\hline
\end{tabular}

All of the interviews were audio-recorded and later transcribed. Each of the transcripts were coded separately and later analyzed for recurring themes across interviews. We also collected relevant artifacts such as stored e-mails, IMs, and files containing traces of communication utilized during the ideation process and analyzed these to verify opinions expressed during the interviews and designers' actual practices. Though the study was qualitative in nature, numbers were reported to highlight the relative significance of an observed behavior.

\section{Study Results}

We report the results from our study of communication surrounding artifacts during ideation activities. We discuss why, with whom and when communication occurs, discuss communication categories, channels utilized, and strategies adopted by the designers for managing communication during ideation.

\subsection{Role of Communication in Ideation Process}

Communication Accounts for more than 50\% of Ideation Activity: Designers deem communication as a core design activity and reported that on an average more than $50 \%$ of early design activity involves communication related to artifacts.Designers communicate to learn about existing artifacts, generate ideas, compare alternatives, and refine and select ideas for further consideration. They engage in communication about the artifacts and utilize the outcomes of communication to guide the design process.Designers leverage artifacts and associated communication to capture the underlyingdesignprocess and believe that artifacts or communication alone fails to adequately represent their process. As a result, designers want to capture artifacts and relatedcommunication together as it facilitates reuse, in line with findings reported in[19]. Existing technology do not support capturing and management of artifacts and communication in one space, resulting in disassociation between these two design components. This forces designers to come up with ad-hoc strategies to link artifacts and communication for effective utilization. 
Though designers mentioned engaging in communication throughout the design process; they believed the impact of communication is most significant during the early phases when they are striving to come up with conceptual ideas. We observed designers' workspaces showcasing different types of early design materials, where communication is captured and highlighted in terms of feedback on post-its, annotation on sketches, list of requirements, etc. Figure 1 presents one such workspace where artifacts and related communication are displayed in the idea space to guide the design process. Idea spaces showcasing artifacts and associated communication is typical in all the designers' physical workspaces, indicating a need for better integration of these two design components in the design support tools.

Communication Helps Resolve "Designer's Block": Fourteen out of fifteen designers interviewed mentioned engaging in communication as a method of getting past "designer's block." Designers consider communication not only necessary but also as a fail-safe method for removing the block. To quote two designers:

"There's a point when you reach your road block in the design process and you need fresh perspective, you need someone to ask "hey, what do you think?" That happens a lot." [P4]

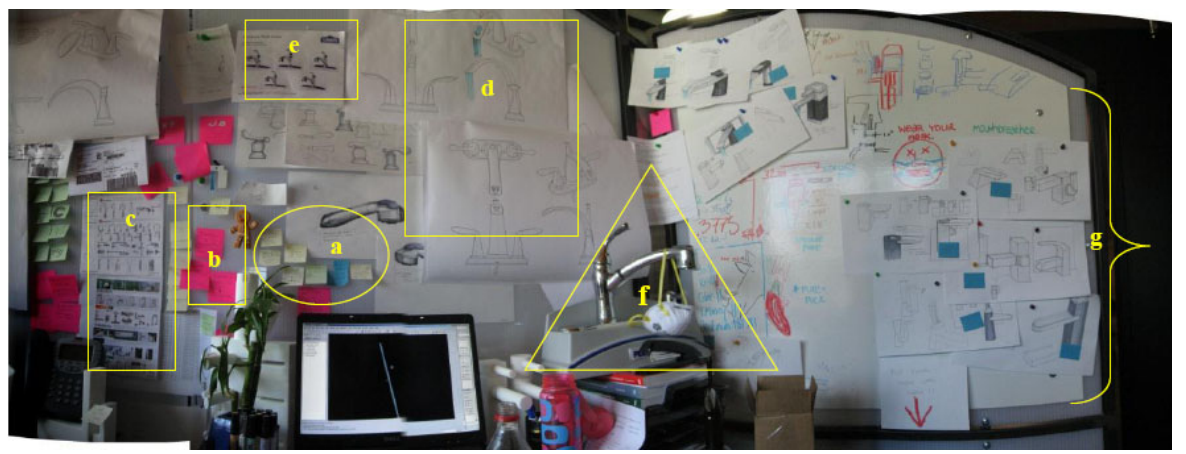

Fig. 1. Different types of early design materials: (a) ideas on post-its; (b) feedback on ideas; (c)materials collected from the Web; (d) prototype sketches; (e) product images; (f) physical products; and (g) notes from brainstorming sessions; displayed in a designers' workspace. Artifacts and communication are captured in close physical proximity to assist in ideation.

"Everyone gets stuck no matter how creative or how good you are. And that's when other designers will come out and they might be mean about it, but I like to think that everyone won't be blunt about it. I'll post (in Web forums) to get help." [P10]

\subsection{Communication in Different Phases of Early Design Activity}

Information Seeking - Personal Communication is Preferable to Web Search: The first step during ideation is to collect information for problem formulation. Designers communicate with other designers, clients, and end users to better situate the design problem, to collect information on the product and similar projects, and to collect requirements from clients. Designers also collect information from office re- 
pository, the Web, books and magazines. While Web and other sources allow quick access to information, opportunity to learn from other designers' experience makes personal communication the most preferred method for collecting information. Designers search technical information on the Web, but communicate with other designers for collecting subjective information (e.g., feedback, rationale). See figure 2 for most utilized sources of information during ideation activities. Designers also communicate with other designers whom they believe to be invaluable sources of design knowledge, which can't be attained from any other sources. This preference is exemplified by the following two quotes:

"Usually I'll gravitate towards who I know first. I'll start from my old colleagues, I'll ask my whole group, and then do a search on the Internet. If I am looking for some technical information, I'll first search in the Internet, but if I get really stuck, I'll ask people more about it." [P8]

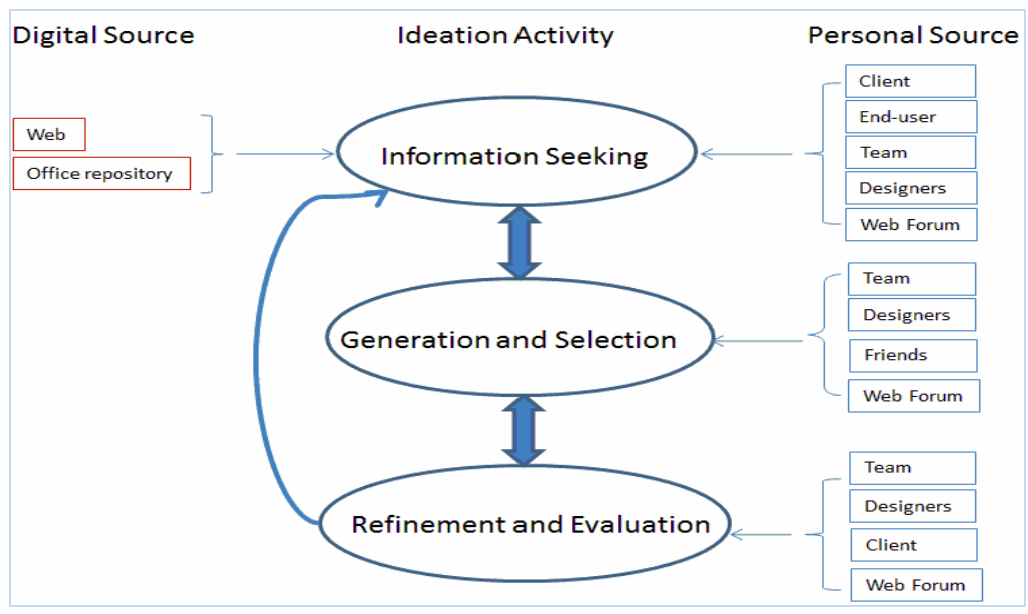

Fig. 2. Information sources utilized during ideation activities. Sources are listed in order of preference (client is preferred than end-users for information seeking).

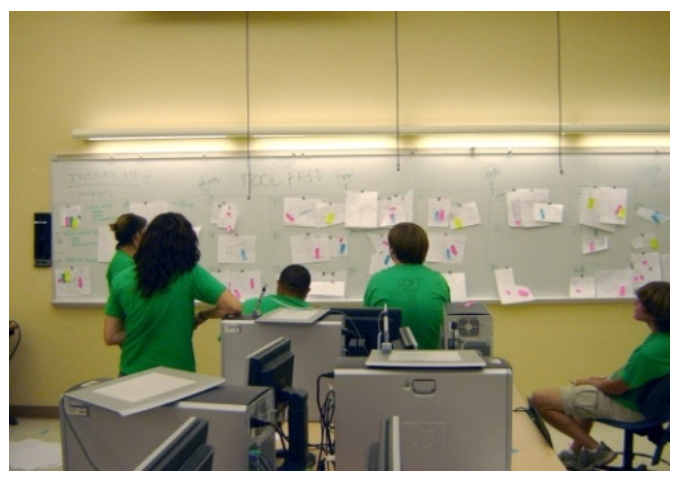

Fig.3. Group communication session targeted to share information and generate ideas. Early ideas are posted on a white board for discussion and selection. 
"There is so much knowledge on gaming culture and you can't find it in Google or you can read so many books, but still not find the answer. You have 100 200 people who have these small pockets of knowledge and together it's pretty formidable." [P7]

\section{Generation and Selection of Ideas - Integration of Artifacts and Related} Communication is Imperative: During ideation, designers mentioned engaging in frequent communication with others designers, especially when they get stuck and need fresh perspectives. After initial research, designers engage in brainstorming sessions to share information, to narrow down the problem space, and to generate, refine, and select ideas for refinement. Research on early design also highlighted the importance of team meetings and brainstorming on the innovation process [3].

Figure 3 depicts one such session where members of a group are engaged in ideation. While these sessions indicate close relationship between artifacts and communication, existing tools support capturing either artifacts or communication. As a result, relationships between artifacts and communications are lost. However, we observed designers making an effort to preserve these relationships by capturing the outcomes of communications using notes and e-mails to themselves and storing these along with related artifacts. When asked, designers mentioned that artifacts and communications separately fail to capture the process and rationale for generating and selecting ideas, and the value lies in capturing these two components together. Eleven out of the fifteen designers mentioned creating notes and/or taking pictures of the white boards filled with discussion points to preserve outcomes of the communication with the other design materials. This indicates a need for tools that would better connect communication outcomes with the other design materials.

Refinement and Evaluation through Feedback: Another type of communication prevalent in early design is feedback, which allows designers to refine, improve and validate their ideas. A significant part of early design communication is targeted to receive and provide feedback. Designers also utilize feedback to learn about potential weakness and strength of their ideas and to examine and enhance the quality of ideas. The most cited reason for feedback request is to receive a fresh and alternative perspective on an idea.One designer summarized this behavior nicely:

"If I'm on my own doing a project I get too close to it to see other issues or problems. If someone has a fresh look at it they would say does that really work or how does that work. I would never think of that. Someone hasn't been staring at the same thing. Play devil's advocate. (And ask) Would that really work?" [P3]

All of the designers participating in ourstudy mentioned requesting feedback on their ideas, two of the fifteen mentioned refining the ideas before requesting any feedback, and four mentioned requesting feedback throughout the ideation process.

\subsection{Medium of Communication - A Shift towards Digital Channels}

Communication occurs throughout the ideation process; it begins even before designers start generating ideas. Designers utilize different communication channels (faceto-face conversations, over phone, via e-mail and IM) based on the underlying design activity and the phase of the design. Figure 4 presents a distribution of utilized and preferred communication channel - indicating a shift towards the digital channels. 
While face-to-face communication is considered imperative, digital channels, especially e-mail is the most utilized channel to initiate, manage, and create a record of communication outcomes.Interestingly, $60 \%$ of the designers mentioned following up with e-mails after face-to-face or phone conversations. The capability of including images of artifacts along with the discussion and ease of sharing and access make email their preferred choice for communication. One designer stated:

"I hang on to all the e-mails. I actually try to take notes on what people say. I take notes in text files and keep them in the same folder like the Photoshop files." [P9]

\subsection{Communication Capture and Management}

Communication Capture: Designers make genuine efforts to capture outcomes of Communication for a number of reasons. Communication in terms of design decisions, feedback, and thoughts on ideas allows designers to present their rationale to

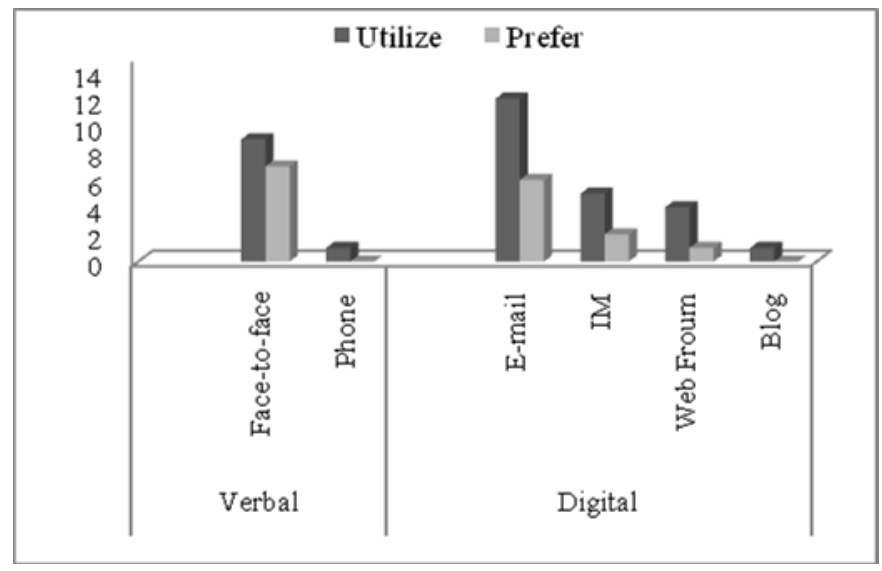

Fig. 4. Distribution of communication channel use. X-axis represents channels and Y-axis represents number of designers. All but one participant reported utilizing multiple channels.

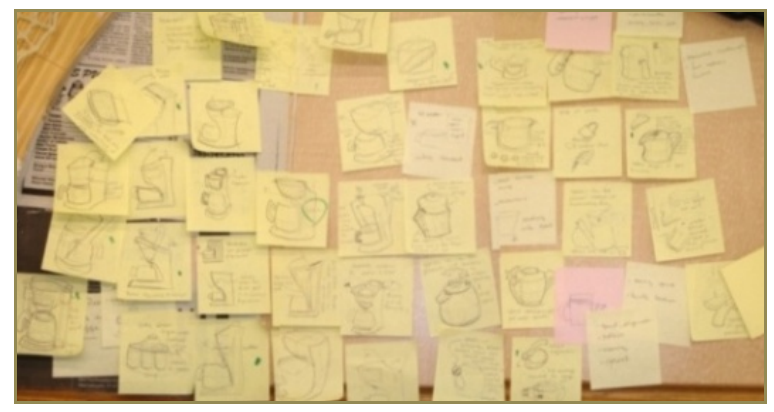

Fig. 5. Anidea space of one designer. Post-its contain annotated sketches displaying comments, feedback, and rationale for each design choice. 
the clients and others. Designers consider capturing and presenting feedback on an idea and rationale for selecting a direction as a way of validating their design. To quote a designer:

"When somebody comes and you show them your final product, and they just think that you put those things together somehow magically but actually there were lot of iteration, there's lot of thinking that went behind it, and it's kind of a way to show "here's actually what I was thinking."'[P4]

Designers also try to capture communication outcomes to select the best idea from a number of possible candidates. To some designers selecting the best direction is often as challenging as coming up with numerous ideas [19]. Designers tend to capture early ideas and associated feedback on post-its and use these to analyze alternate directions. Figure 5 shows idea space for a designer where she accumulated her ideas along with feedback received and rationale behind her design choices. She utilized this space not only for selecting a direction but also for showing the client and others her process. Spaces such as this are common in the design workspaces, illustrating the need for better integration of artifacts and relevant communication.

Communication Management: Existing technology poses challenges in the way designers try to manage communication surrounding artifacts. Designers want to record these communications not only for using in the ongoing project, but also as a reference for future projects. Especially, communication with clients in terms of requirements, feedback, and their preferences are stored for potential future projects. Figure 6presents one such text file that one designer (P9) created to record all the feedback received for an ongoing project. This file is used to keep track of the suggestions from other designers and his reaction to these suggestions.However, the designer expressed frustration as these communications are disconnected from each other and from the artifacts and he has to invest a great deal of effort to create a single space to combine the outcomes of communication and resulting artifacts.

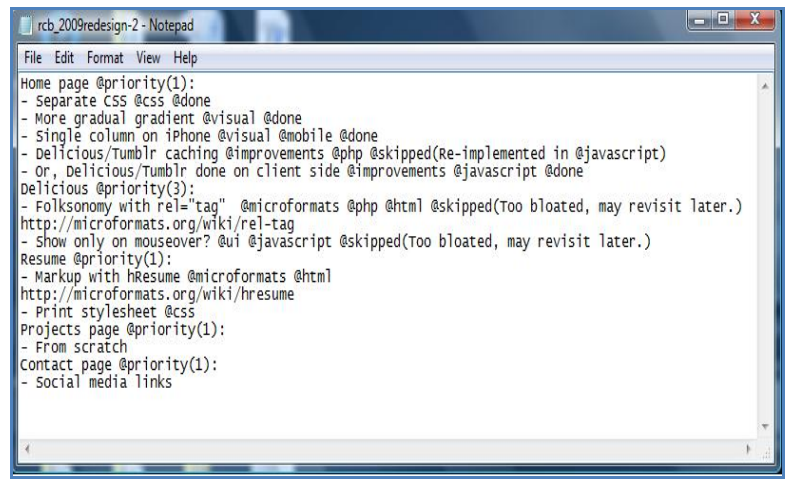

Fig. 6. Combining summaries of feedback received from various communication channels in a single text file 
Artifacts are typically stored in directory-based file management systems while communication is stored as e-mail archives, chat logs, voice mails, etc. The separation of these two types of design materials forces designers to adoptad-hoc strategies to connect them together.For example, we observeddesigners investing lots of effort to record all the communication related to an artifact by creating files that combine summaries of communications received from all different channels. Anotherpopular strategy was to mimic the directory structure in e-mail clients to reduce the burden of management (see figure 7). Similar finding has been reported in [25] for organizing materials in the area of software design.

\subsection{Influence of Work Setting: Access, Culture, Ownership and Criticism}

Work setting influences designers' strategies and motivations for communication. Culture of organization, ownership of ideas, fear of criticism, and access to design resources greatly impact designers' experience surrounding communication.

Access to Communication Partners: Designers working in design firms tend to communicate more during the ideation process as they have access to team members and other designers working in the organization. Freelance designers only have access to clients as most of them work alone. Lack of access to other designers and intellectual property issues force them to limit their communication within a selected and trusted group of designers and with clients.Freelance designers often mentioned relying on close friends and family members to bounce ideas off of due to a lack of access to other experienced designers.One freelance designer mentioned consulting his spouse (a non-designer) when faced with challenge during the ideation process.Freelance designers try to utilize online design forums to gain access to other designers who may eventually become trusted communication partners. Every freelance designer mentioned this lack of connection with other designers as one of the key challenges faced during early design phases.

Figure 8 reflects designers' preference for feedback request. For designers working in organizations, team members are the most preferred source $(63 \%)$, while freelancers mentioned clients $(57 \%)$ as their primary source for feedback. This difference is due to the accessibility to other designers, clearly indicated by the fact that designers working in organizations never mentioned clients as a potential source for feedback on early artifacts. However, a fairly large number of designers (43\% and 50\% respectively) prefer to communicate with friends or peers (who are not involved in the project) about artifacts. A significant percentage of freelance designers (29\%, mostly novice) preferred online forums, while none of the designers working in firms considered online forums as useful source for feedback.

Organizational Culture: Organizational culture has been regarded as the primary factor that hinders or promotes communication. Team boundary often discourages communication within an organizational setting as designers feel "less comfortable" communicating and providing feedback to other designers who belong to other design teams. One designer shared his experience with different organizational culture and how it impacted his communication behavior: 


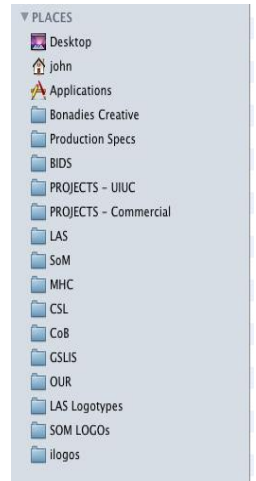

(a) Artifact Space

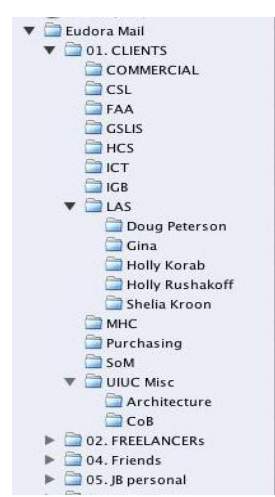

(b) Communication Space

Fig. 7. Managing artifact space and communication space by creating similar structure in the hard drive and e-mail client

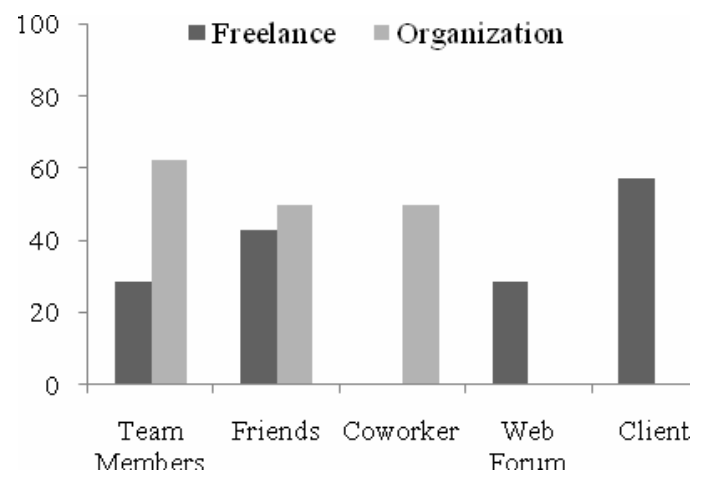

Fig. 8. Designers' preferred group for requesting feedback. Y-axis represents designers' preference $(\%)$. Empty bars indicate there was no preference for the group.

"Many places are hyper competitive and success mattered on how often you won, and mostly jobs will be competitive among a number of people and it was important that you won, and in some places the culture is such that in a way it rewards that behavior. So people stole ideas and it became a territorial thing, in that case you wouldn't share because you couldn't count on the goodwill and good faith...But here the culture is not like that at all as they don't reward behaviors like that. We are all quite comfortable to walk in our buddy's office and share ideas and ask feedback." [P14]

Ownership of Ideas and Fear of Criticism: A large number of designers refrain from communicating early ideas within the organization as they fear that negative or blunt criticism may diminish their enthusiasm for the idea. Competition among team members is also mentioned as one of the reasons why designers feel discouraged to communicate. Even designers working as part of a team tend to delay communication 
until they feel comfortable with the quality of generated artifacts. This behavior is exemplified by the following two quotes:

"Typically you don't communicate as much when you haven't got your idea yet. You don't want to sense that you are stealing from someone else's ideas. You don't want to talk to somebody so that they come up with the idea that you yourself don't feel like it's truly yours. These are the times that I am hesitant to get feedback." [P14]

"I feel that people might criticize things that I am already aware of that need to be changed, so I want to get to a state where I feel like I have done most of, I have contributed most of what I want to do, and then see what other people think about it as opposed to starting something and ask other people before I am done." [P9]

Majority of the freelance designers use online forums for communicating ideas and expressed that lack of control over the audience and the likelihood of receiving negative feedback are their biggest concerns. One designer went as far as boycotting a forum as he considered the feedback posted as negative and discouraging. This behavior is quite common among freelance designers and can be better exemplified by a comment made by P6:

"A place like site2 (pseudonym) forum will discourage me to share information, I think there are some fantastic designers there but between site 2 and site1,itis a very separate group of people when it comes to personalities. (In site2) They do not hesitate to say that you are an idiot or stupid while in sitel its little bit more laid back, little bit softer, and it's not that harsh." [P6]

\subsection{Influence of Expertise: Communication Categories, Partners and Motive}

Level of expertise influences how and why designers communicate, with whom they communicate, and how do they capture and utilize communication in their design.

Communication Categories: While both expert and novice designers mentioned information collection as one of the primary reasons for initiating communication, the types of information that designers request vary depending on designers' level of expertise. Expert designers rarely request technical information from other designers while novices mentioned other designers as the best resource for finding technical information.Expert designers feel socio-psychological pressure of "not knowing enough" and are reluctant to ask for help on technical issues but novice designers consider themselves as learners and feel that they are expected to ask. Expert designers, contrarily, request more for client and product related information to learn more about the design space and from the experience of other designers. Expert designers mentioned that more than $80 \%$ of their communication consists of feedback and discussion about design decisions. On the contrary, a significant part of early communication for novice designers is aimed for generating, comparing, and refining ideas along with reputation building. Figure 9 shows designers' motivations for communication. While both expert and novice designers mentioned communication as a means of collecting information, receiving feedback and reciprocity, novice designers communicate more to receive creative input on ideas than expert designers. 


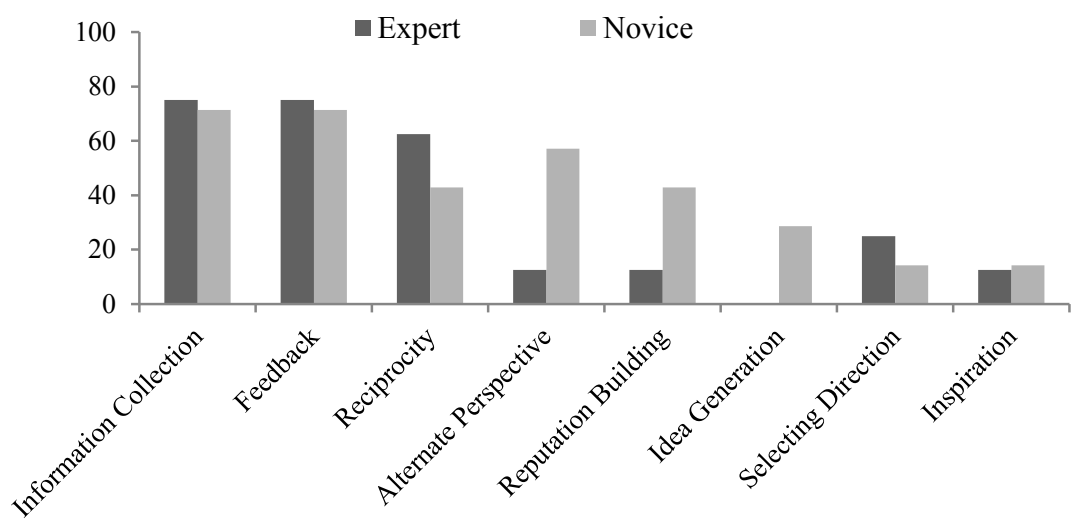

Fig. 9. (Expert vs. Novice) Designers' motivation for engaging in communication. X-axis represents reasons and $\mathrm{Y}$-axis represents the percent of designers who prefer them.

Communication Partners: Finding appropriate communication partners often poses a problem for novice designers. Trust plays a significant role in selecting designers for communication about early ideas, often due to fear of negative criticism and threat of exposing unfinished ideas. While expert designers utilize prior experience to find trusted partners, novice designers often struggle in this process. Novice designers go as far as to study the history of communication of designers (collecting information from colleagues or reviewing past communication) to select the best candidates for communication. Novice designers try to build relationships by posting on design forums and by contacting designers personally. However, these approaches help little asdesigners rarelyafford to invest time to analyze communication history ofindividual designers to find a proper fit. Automated recommender systems can greatly help in the process of finding suitable communication partners.

Utilization of Communication Outcomes: Expert and novice designers' tend to communicate their ideas at different times within the ideation process. Novice designers tend to communicate throughout the ideation process and use the feedback to refine ideas. Expert designers rarely share their initial ideas, waiting till it reaches a certain point where they are either satisfied with the idea or are suffering from "designer's block." Novice designers also show more openness towards harsh feedback as they view it as learningprocess. To quote a designer:

"Experience plays a role (in how you utilize it). If you are young, you ask feedback differently and receive it differently, and you would accept even challenging or disappointing feedback. And you also don't have the confidence to say that I hear you, but I don't buy it. The younger you are the more likely you are to do everything anybody tells you. And having more experience, you learn to understand what is important and what might be ok but not certainly important." [P14] 


\section{Discussion and Design Implications}

In this section we discuss implications derivedfromlessons from this study. The implications presented are not exhaustive, but they offer guidance for better connecting communication and relevant artifacts and removing barriers for engaging in communication for ideation.

\subsection{Integrating Communication Traces with Artifacts}

Artifacts and surrounding communications are the two main components of ideation process. Communication traces help designers recall their design choices and convey their process and rationale to others. However, the disassociation of artifacts from communication makes it challenging to effectively utilize communication outcomes during the ideation process. Lack of suitable mechanisms for connecting communication and artifacts for long term management makes it extremely difficult to access, retrieve and utilize communication outcomes from ongoing or past projects. Every designer studied expressed frustration as this disconnection forces them to develop ad-hoc strategies and discourages them to revisit existing design knowledge.

Linking communication channels, such as, e-mail and IM, with project spaces will allow better integration of artifacts and related communication. When designers communicate through these channels, options should be provided to link the artifacts with the communications. Similarly, designers should be able to access relevant communication from their artifact spaces. Integrating plug-ins in the e-mail clients (e.g., Outlook) to create a bidirectional channel between the communication space and artifact space would allow designers to make this connection. Linking communications with artifacts would require some effort from the designer, but we believe that designers would be willing to invest this effort if it allows better retrievability.

\subsection{Categorizing and Organizing Communication}

Different types of communication influence the design process differently. While some communication allows the designer to select the best direction, others assist to improve the quality of artifacts. Some types of communication have value that extends well beyond the lifetime of a project, while others are only important during an ongoing design. Designers want to categorize and organize communication in a manner that facilitates effective access, retrieval and utilization. However, categorization of various types of communication introduces overhead in the design process, for which designers want to (and can) invest little effort.

Creating a vocabulary for capturing and categorizing different types of communication with related artifacts (e.g., requirement, user preference, design decision, and feedback) would allow effective access and retrieval. Incorporating default spaces (folders for client communication, feedback, rationale) for different categories of communication in the artifact space could reduce the burden of organization on the designer. Alternatively, tags could be utilized to associate different types of design communication with appropriate categories. These tags could be integrated in the e-mail and IM clients and designers could attach one or more tags to link the 
communication (and relevant artifacts) to the appropriate category. Selecting a specific tag would provide access to all materials linked to that category.

\subsection{Aggregate Communication from Multiple Channels}

Designers engage in communication throughout the ideation process and obtain valuable information from various channels, such as face-to-face and phone conversations, e-mail, IM, online forums, etc. Lack of support for aggregation of communications obtained from various channels makes it difficult and time-demanding for designers to effectively utilize communication in the design process.

While it may not be possible to capture verbal conversations without any effort from the designer, communication that occurs through digital channels can be aggregated automatically. To facilitate lightweight capture of verbal communication, artifact spaces should incorporate free-form text entry spaces such as notes, which will allow designers to add communication outcomes with the artifacts. Allowing aggregation of communication obtained from various digital channels (e-mail, IM, forum) along with relevant design artifacts through lightweight linking could provide a solution to this problem. Add-ons in the e-mail and IM interfaces could be used to link pertinent communication to an artifact. Add-ons could be integrated in the online forums to allow designers to connect selected communications to the artifact space. Ideally, the artifact space should be connected to all communication channels and should provide support for linking communications received from various channels.

\subsection{Support Finding Communication Partners by Integrating Interaction, Preference, and Availability Information}

Designers almost always prefer to communicate with designers who can offer them valuable insight to improve the quality of their design. Expert designers often have access to suitable communication partners (other designers whom they deem fit), but it is difficult for novice and freelance designers to find appropriate communication partners. On the other hand, it can also become demanding for known experts in an area to accept communication (feedback) request from many designers.

A possible solution can be allowing designers to include communication preference and availability in their organization and/or online profile (in intra-networks, wikis). A visual history of projects they have worked on, clients they have worked for, their area of expertise and interest can be attached to their profile and also can be utilized for suggesting communication partner against a designers' query. For an online public setting, past communication can be analyzed including posts they have initiated and responded to. Designers' search for communication partners could be used along with their profile and interaction history to recommend a list of potential communication partners. Designers could select other designers from the suggested list or by analyzing the profiles that best meet their communication needs.

\section{Conclusion}

Communication surrounding artifacts is one of the key activities during the ideation process. Though designers utilize communication and design artifacts simultaneously 
throughout the ideation process, existing research on design focus on these components separately; resulting in tools which make effective utilization of artifacts and related communication challenging. Better technology can assist to integrate artifacts with communication; however, in-depth understanding of communication practices in relation to artifacts is critical before building such systems.

This paper has contributed deeper understanding of communication practices in relation to artifacts during the ideation process. Our findings indicate communication outcomes greatly influence the generation, refinement, and selection of ideas during the early design stages. We also identify organizational and social parameters that influence designers' communication practice. We observed designers making genuine efforts, but struggling to capture communication outcomes with relevant artifacts, indicating a need for better design support tools. We offer guidelines that can be utilized to design new systems or to extend existing tools to better support the interconnection of communication with relevant design artifacts.

\section{References}

1. Eckert, C., Stacey, M.: Sources of inspiration: a language of design. Design Studies 21(5), 523-538 (2000)

2. Sonnenwald, D.H.: Communication roles that support collaboration during the design process. Design Studies 17(3), 277-301 (1996)

3. Sutton, R.I., Hargadon, A.: Brainstorming Groups in Context: Effectiveness in a Product Design Firm. Administrative Science Quarterly (1996)

4. Bonnardel, N.: Creativity in Design Activities: The Role of Analogies in a Constrained Cognitive Environment. In: Proc. Creativity \& Cognition, pp. 158-165 (1999)

5. Garner, S., McDonagh, D.: Problem Interpretation and Resolution via Visual Stimuli: The Use of 'Mood Boards' in Design Education. Art \& Design Education 20(1) (2001)

6. Büscher, M., Kompast, M., Lainer, R., Wagner, I.: The Architect's Wunderkammer: Aesthetic Pleasure and Engagement in Electronic Spaces. Digital Creativity 10(1), 1-17 (1999)

7. Landay, J.A., Myers, B.A.: Interactive Sketching for the Early Stages of User Interface Design. In: Proc. CHI, pp. 43-50 (1995)

8. Regenbrecht, H., Haller, M., Hauber, J., Billinghurst, M.: Carpeno: interfacing remote collaborative virtual environments with table-top interaction. Virtual Reality 10(2), 95-107 (2006)

9. Stewart, J., Bederson, B.B., Druin, A.: Single display groupware: A model for co-present collaboration. In: Proc. CHI, pp. 286-293 (1999)

10. Conklin, E.J., Burgess-Yakemovic, K.: A Process-Oriented Approach to Design Rationale. Journal of HCI 6, 357-391 (1996)

11. Lee, J.: SIBYL: a tool for managing group design rationale. In: Proc. CSCW, pp. 79-92 (1990)

12. Chiu, M.L.: An organizational view of design communication in design collaboration. Design Studies 23(2), 187-210 (2002)

13. Kraut, R.E., Fish, R.S., Root, R.W., Chalfonte, B.L.: Informal communication in organizations: Form, function, and technology. Morgan Kaufmann, CA (1993)

14. Nardi, B.A., Whittaker, S.: The Place of Face-to-Face Communication in Distributed Work. MIT Press, Cambridge (2002) 
15. Eckert, C., Stacey, M.: Dimensions of Communication in Design. In: International Conference on Engineering Design (2001)

16. Stempfle, J., Badke-Schaub, P.: Thinking in design teams - an analysis of team communication. Design Studies 23(5), 473-496 (2002)

17. Herring, S.R., Chang, C.-C., Krantzler, J., Bailey, B.P.: Getting inspired!: understanding how and why examples are used in creative design practice. In: Proc. CHI, pp. 87-96 (2009)

18. Kolodner, J.: Improving Human Decision Making Through Case-based Decision Aiding. AI Magazine, 52-68 (1991)

19. Sharmin, M., Bailey, B.P., Coats, C., Hamilton, K.: Understanding knowledge management practices for early design activity and its implications for reuse. In: Proc. CHI, pp. 2367-2376 (2009)

20. Klemmer, S.R., Newman, M.W., Farrell, R., Bilezikjian, M., Landay, J.A.:The designers' outpost: a tangible interface for collaborative web site. In: Proc. UIST, pp. 1-10 (2001)

21. Klemmer, S.R., Thomsen, M., Phelps-Goodman, E., Lee, R., Landay, J.A.: Where Do Web Sites Come From? Capturing and Interacting With Design History. In: Proc. CHI, pp. 1-8 (2002)

22. Conklin, J., Begeman, M.L.: gIBIS: A Hypertext Tool for Team Design Deliberation. In: Proc. Hypertext, pp. 247-251 (1987)

23. Reeves, B., Shipman, F.: Supporting Communication between Designers with ArtifactCentered Evolving Information Spaces. In: Proc. CSCW, pp. 394-401 (1992)

24. Arias, E., Eden, H., Fisher, G.: Enhancing communication, facilitating shared understanding, and creating better artifacts by integrating physical and computational media for design. In: Proc. DIS, pp. 1-12 (1997)

25. Atwood, M.E., Burns, B., Gairing, D., Girgensohn, A., Lee, A., Turner, T., Alteras-Webb, S., Zimmermann, B.: Facilitating Communication in Software Development. In: Proc. DIS, pp. 65-73 (1995)

26. Lucero, A., Aliakseyeu, D., Martens, J-B.: Funky Wall: Presenting Mood Boards Using Gesture, Speech and Visuals. In: Proc. AVI, pp. 425-428 (2008)

27. Tang, J.C., Leifer, L.J.: A Framework for understanding the workspace activity of design teams. In: Proc. CSCW (1988) 